\title{
Single-molecule fluorescence studies on DNA looping
}

\author{
Jiyoun Jeong ${ }^{\mathrm{a}}$, Tung T. Le ${ }^{\mathrm{a}}$, Harold D. Kim ${ }^{\mathrm{a}, *}$ \\ ${ }^{a}$ School of Physics, Georgia Institute of Technology, 837 State Street, Atlanta 30332, USA
}

\begin{abstract}
Structure and dynamics of DNA impact how the genetic code is processed and maintained. In addition to its biological importance, DNA has been utilized as building blocks of various nanomachines and nanostructures. Thus, understanding the physical properties of DNA is of fundamental importance to basic sciences and engineering applications. DNA can undergo various physical changes. Among them, DNA looping is unique in that it can bring two distal sites together, and thus can be used to mediate interactions over long distances. In this paper, we introduce a FRET-based experimental tool to study DNA looping at the single molecule level. We explain the connection between experimental measurables and a theoretical concept known as the $\mathrm{J}$ factor with the intent of raising awareness of subtle theoretical details that should be considered when drawing conclusions. We also explore DNA looping-assisted protein diffusion mechanism called intersegmental transfer using protein induced fluorescence enhancement (PIFE). We present some preliminary results and future outlooks.
\end{abstract}

Keywords: DNA looping, J factor, single-molecule fluorescence, FRET, PIFE

\section{Introduction}

Double-stranded DNA is a complex macromolecule composed of many different kinds of atoms and layers of interactions. It is also highly dynamic with diverse modes of motion including bending, twisting, and stretching[1]. Despite this complexity, mechanical properties of DNA at large length scales can be well-described by the worm-like chain (WLC) model. According to this model, directional change in the tangent to the chain contour is associated with bending energy. The stiffness of double-stranded DNA (referred to as DNA unless stated otherwise) can be described by a length scale over which the direction of the tangent tends to persist in spite of thermal fluctuations. This length scale is known as the persistence length and at room temperature is estimated to be $\sim 50 \mathrm{~nm}(147 \mathrm{bp})$ for DNA by various methods $[2,3,4,5]$. Using this value, the elastic energy cost of deforming $\sim 50 \mathrm{~nm}$

\footnotetext{
*I am the corresponding author

Email addresses: jjeong41@gatech.edu (Jiyoun Jeong), tt143@cornell.edu (Tung T. Le), harold.kim@physics.gatech.edu (Harold D. Kim)

URL: https://sites.google.com/site/haroldkimlab (Harold D. Kim)
} 
DNA into a closed circle is estimated to be $\sim 20$ times the thermal energy $\left(k_{B} T\right)$. Therefore, looping of DNA shorter than one persistence length, which will be hereafter termed "short DNA", is expected to be thermodynamically rare.

Nonetheless, looping of short DNA DNA looping is often associated with important biological processes $[6,7]$. In transcriptional regulation, transcription factors can bridge two different sites on the DNA when the intervening DNA toops out forms a loop $[8,9,10]$. In eukaryotes, nucleosomes, the packaging units of the genomic DNA, contain about 1.7 turns of $\sim 146 \mathrm{bp}$-long DNA[11]. The protein shell of a virus, typically $30 \mathrm{~nm}$ to $100 \mathrm{~nm}$, can accommodate $10 \mu \mathrm{m}$-long genomic DNA because of strong DNA bending[12]. Looped genes and nucleosome favoring sequences are correlated with high DNA flexibility to some degree[13, 14], which further implicates the role of spontaneous strong DNA bending in genome biology. DNA looping is not only a physiologically important mechanism that contributes to DNA packaging and gene regulation, but may also serve as a mechanism to facilitate transporting proteins over long distances. In a mechanism known as intersegmental transfer, a protein bound to DNA at one site can be transferred to another remote site when the two sites are juxtaposed. In vitro evidences show protein transfer between different DNA molecules depends on the concentration of DNA molecules carrying possible binding sites $[15,16,17,18,19]$, which strongly hint at the possibility of intersegmental transfer even within the same DNA.

To study thermodynamics of DNA bending, end-to-end ligation of linear DNA has been widely used[20]. In this cyclization assay, the DNA molecule has complementary singlestranded overhangs called "sticky ends." By thermal excitation, the sticky ends will approach each other within a short annealing range with a small, but nonzero probability. DNA ligase is added to convert the transient, end-to-end annealed loops into covalently closed circles. As a side reaction, different DNA molecules are also ligated to each other to form dimers (and multimers). The amounts of DNA circles and dimers are quantified by gel electrophoresis as a function of time, from which the apparent rate constants for cyclization and dimerization can be extracted. The first-order cyclization rate constant divided by the second-order dimerization rate constant yields a quantity in units of molar concentration. This quantity, known as the $\mathrm{J}$ factor[21], can be interpreted as the effective concentration of one end of a chain in the vicinity of the other end. For elastic chains, the $\mathrm{J}$ factor is expected to decrease as the chain becomes shorter because of the bending energy cost.

Although the DNA cyclization assay successfully validated the WLC model prediction with long DNA[22], it has serious limitations when it comes to looping of short DNA[23, 24]. First, because cyclization is rare for short DNA, the ligation reaction is dominated by dimerization, and the interpretation of the result becomes challenging. It was shown that high ligase concentration can bias the apparent dimerization constant[24], thus causing an overestimation of the $\mathrm{J}$ factor at short length scales. Another drawback of the assay is the ligase itself, which nonspecifically binds DNA, loses activity over time during reaction, and only works in a limited range of salt conditions and temperature.

Recently, researchers began to use single-molecule fluorescence resonance energy transfer (smFRET) to tackle questions on DNA bendability[25, 26, 27, 28]. By immobilizing DNA molecules with 7-10 base long sticky ends on a glass surface, one can monitor reversible 
looping and unlooping events in real time under a microscope without competing dimerization as in bulk cyclization[27]. Moreover, because end-to-end annealed DNA is torsionally relaxed around the nicks, the looping equilibrium is not influenced by torsional rigidity of DNA. Unlike the irreversible ligase-dependent cyclization assay, the smFRET assay also gives access to the unlooping transition, which can give us insights into the energetics of sharply bent dsDNA[28].

DNA looping is not only a powerful tool to study bendability of DNA, but also a physiologically important mechanism that contributes to DNA packaging and gene regulation. DNA looping may also play a dynamic role in transporting proteins over long distances. In a mechanism known as intersegmental transfer, a protein bound to DNA at one site can be transferred to another remote site when the two sites are juxtaposed. In vitro evidences ef protein transfer between different DNA molecules in a concentration dependent manner $[15,16,17,18,19]$ strongly hint at the possibility of intersegmental transfer even within the same DNA. Despite many studies on DNA looping, the role of DNA looping in facilitating protein diffusion has not been directly addressed. Recently, looping-mediated intersegmental transfer of proteins has been inferred based on enzymatic modification of DNA at two distal sites $[29,30]$. Also, intersegmental transfer within the same single-stranded DNA (ssDNA) has been demonstrated from the dependence of protein diffusion rate on DNA tension[31]. However, how much intersegmental transfer can accelerate target search kinetics has not been measured. It is also unclear how common this mechanism is among different classes of DNA binding proteins. In this regard, a simple experimental method that produces a direct readout for intersegmental transfer and is applicable to a wide range of DNA binding proteins with minimal modification is highly desirable.

In this article, we explain two single-molecule experiments that can be used to investigate the biophysics of DNA looping and its influence on DNA-protein recognition. First, we summarize a FRET-based single-molecule looping assay that we previously used to study thermodynamics of DNA looping. Second, we introduce a new method based on PIFE (Protein-Induced Fluorecence Enhancement) to investigate DNA-looping-mediated intersegmental transfer of a DNA binding protein.

\section{Theory}

\subsection{The wormlike chain $(W L C)$ model}

What confers double-stranded DNA its rigidity is the stacking interaction, that is, the tendency for adjacent base pairs to stack in a parallel fashion. The most stable conformation of DNA resembles a spiral staircase where steps are analogous to base pairs (Fig. 1B). This analogy is also used as a means to coarse-grain DNA. At this level of coarse-graining, DNA conformation requires three translational (rise, slide, shift) and three orientational (roll, tilt and twist) parameters to define the relative geometry of adjacent base pair steps. These parameters are called base-pair parameters and differ among all ten unique dinucleotide steps. To model bending fluctuations of DNA, translational parameters are usually ignored.

Dinucleotide bending is highly anisotropic in that it prefers to roll (rotation around the long axis of a base pair) than tilt (around the short axis). But since the roll and tilt 


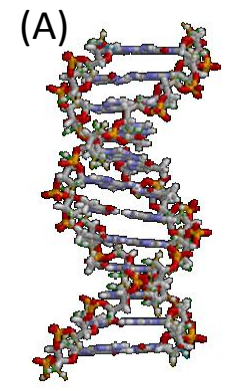

(B)

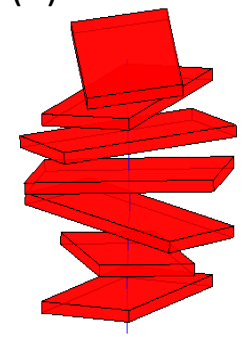

(C)

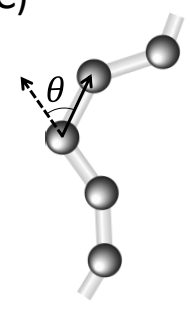

Figure 1: Coarse-graining DNA. (A) The structure of B-DNA at the atomistic level. (B) Rigid base pair model of DNA. Each red slab represents a base pair. (C) Ball-stick representation of DNA. In this discretized model, bending can only occur between sticks around each ball. The length of each stick is equal to the rise per base pair $(0.34 \mathrm{~nm})$. In the wormlike chain (WLC) model, the bending energy at each ball (mass point) is proportional to the square of the angle $(\theta)$ formed by the two adjacent sticks.

axes rotate due to the helical twist, such anisotropic effect ould average averages out over several helical periods. Therefore, to model bending of dsDNA that has a random sequence and is several helical periods long, assigning an isotropic, uniform bending rigidity to each dinucleotide ould suffice is sufficient. DNA can thus be Thus, DNA is described by the ball-stick representation, where the ball roughly corresponds to a base pair, and the stick is a massless, inextensible link whose length $(a)$ is equal to the rise $(0.34 \mathrm{~nm})$ of $\mathrm{B}-\mathrm{DNA}$ (Fig. 1C).

At this level of coarse-graining, there are only two angular degrees of freedom between adjacent links: $\theta$ and $\phi . \theta$ is the difference in the tangent angle between two adjacent links, and $\phi$ is the dihedral angle or the azimuthal angle in a local reference frame. If there is no energy associated with these angular motions, the chain is a freely jointed chain. If energy is associated with $\theta$ only, it is called a semiflexible or wormlike chain (WLC). If energy is associated with both angles, it is called a twisted wormlike chain. For WLC, the bending energy for each monomer is given as a quadratic function of $\theta$

$$
E_{\text {bend }}(\theta)=\kappa \theta^{2}
$$

where $\kappa$ is the rigidity constant. This rigidity coefficient is related to statistical parameter ealled the persistence length $\left(L_{p}\right) . L_{p}$ is the length scale over which the tangent vector to the chain contour persists in a similar direction. Therefore, $L_{p}$ not only depends on the intrinsic rigidity of the chain, but also on temperature. Thus, An equivalent expression of the bending energy in terms of the persistence length is

$$
E_{\text {bend }}(\theta)=\frac{k_{B} T}{2}\left(\frac{L_{p}}{a}\right) \theta^{2}
$$

where $a$ is the monomer length of the chain. $L_{p}$ has been measured using different methods in various conditions. The values can vary widely[32], but the consensus value for modeling purpose is around $50 \mathrm{~nm}[33]$. 


\section{2. $J$ factor calculation}

The two ends of a DNA molecule can approach each other due to thermal excitation, the probability of which is given by the Boltzmann factor. Without losing generality, we can fix one end of the chain at the origin to describe the extension of the chain with one position vector $\mathbf{r}$ of the free end of the chain. In equilibrium, the position vectors of the free end constitute the probability distribution or probability density function $P(\mathbf{r})$. Because the chain is inextensible, $P(\mathbf{r})$ is zero beyond one contour length $(L=N a)$ away from the origin. Therefore $P(\mathbf{r})$ is normalized over the spherical volume of radius $L$ :

$$
\int_{|\mathbf{r}| \leq L} P(\mathbf{r}) d^{3} \mathbf{r}=1
$$

For a free particle in the same spherical volume, $P(\mathbf{r})$ would be constant, equal to $1 / \frac{4}{3} \pi L^{3}$. For a WLC like DNA, $P(\mathbf{r})$ depends only on the magnitude of $\mathbf{r}$. Hence, we can use the scalar end-to-end distance $(r)$ to specify the macrostate of the chain. $P(r)$ can be calculated either analytically or numerically[34]. Examples of $P(r)$ are shown in Fig. 2A for DNA of different lengths. The free energy of this macrostate $(A(r))$ is related to the radial probability distribution $P(r) 4 \pi r^{2}$ :

$$
\frac{e^{-A(r) / k_{B} T}}{Z}=P(r) 4 \pi r^{2},
$$

where $Z$ is the normalization constant (or the total partition function). Therefore, $A(r)$ is given by

$$
\frac{A(r)}{k_{B} T}=\log \left(\frac{1}{4 \pi r^{2} Z P(r)}\right) .
$$

This relationship is useful for depicting the free energy landscape of any transition associated with the change in the end-to-end distance $r$.

We can call a chain conformation as looped when the two ends are very close to each other. Mathematically, any conformation with the end-to-end distance less than some small threshold $r_{0}$ is a looped conformation. In the limit when $r \rightarrow 0, P(r)$ tends to a finite value. In chemistry and biology, it is more convenient to describe this probability density in terms of molarity. Converting the unit of $P(r \rightarrow 0)$ from number density to molar concentration give us a quantity known as the $\mathrm{J}$ factor.

$$
J \equiv \lim _{r \rightarrow 0} P(r)\left[\frac{\text { moles }}{\mathrm{L}}\right] .
$$

Alternatively, the $\mathrm{J}$ factor can be thought of as the average concentration of one end in an infinitesimally small spherical volume around the other end,

$$
J \equiv \lim _{r_{0} \rightarrow 0} \frac{\int_{r=0}^{r_{0}} P(r) 4 \pi r^{2} d r}{\frac{4}{3} \pi r_{0}^{3}}\left[\frac{\text { moles }}{\mathrm{L}}\right] .
$$

The analytical formulae for the J factor of a homogeneous WLC were derived by Shimada and Yamakawa under different end-to-end constraints[35]. The result without angular constraints 
at the ends is plotted in Fig. 2B. The $\mathrm{J}$ factor is a nonmonotonic function of the chain length. At small lengths, the $\mathrm{J}$ factor is dominated by energy, whereas at large lengths, by entropy. Thus, the J factor peaks near $500 \mathrm{bp}$ (top, Fig. 2B), which indicates that sites that are 500-bp apart have the highest probability of juxtaposition. The $\mathrm{J}$ factor changes steeply at lengths below $100 \mathrm{bp}$, which is better appreciated on a semilog plot (bottom, Fig. 2B). The absolute $\mathrm{J}$ factor values and the peak position slightly change when juxtaposition of two interior points of the chain is considered, but the overall trend remains similar[36].

(A)

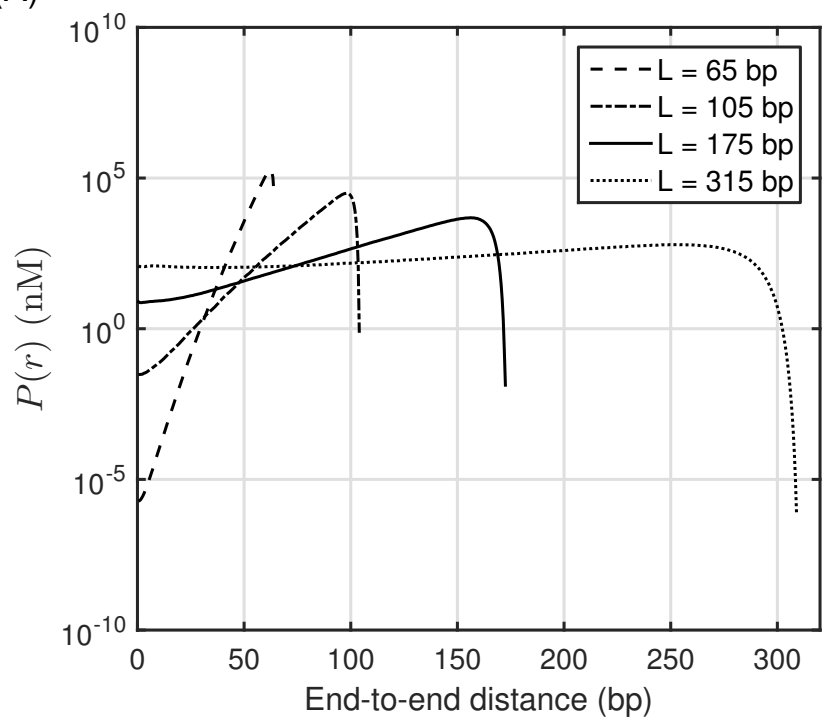

(B)
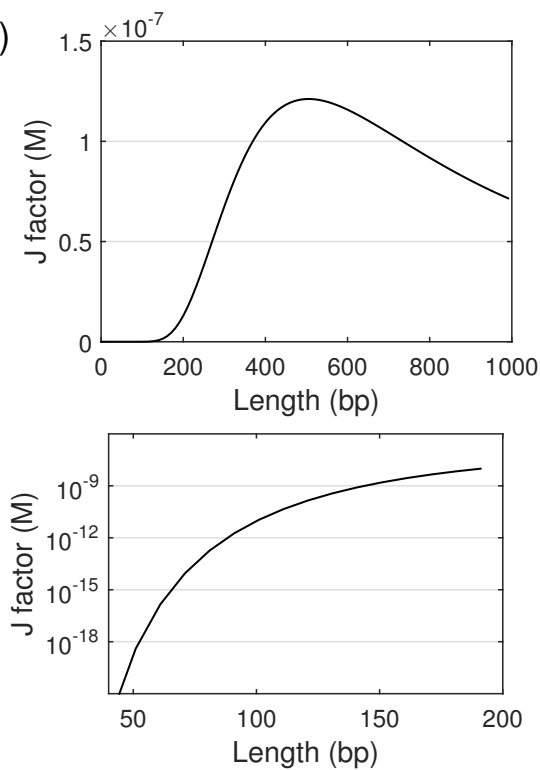

Figure 2: Probability density function and J factor. (A) Probability density function $P(r)$ for different lengths of DNA. These functions are obtained by the simulation method described in 2.2. Because DNA is modeled as an inextensible chain, $P(r)$ drops off near the contour length of the chain. As the length becomes shorter, $P(r)$ approaches the y-axis at a steeper angle. Where these curves cross the y-axis are equal to the J factors. (B) J factor vs. length. These are calculated using the formula[37] originally derived by Shimada and Yamakawa[35] using the persistence length of $50 \mathrm{~nm}$. The top plot shows the J factor on a linear axis over a wide range of DNA lengths. The bottom plot is focused on the controversial region of the relationship on a $\log$ y-axis.

To include anisotropic, asymmetric, or nonuniform bendability and curvature, it is necessary to obtain the $\mathrm{J}$ factor by simulation. The goal of the simulation is to generate a set of chain conformations in thermal equilibrium, which is the canonical ensemble in thermodynamics. The simplest method to perform this task is the Gaussian sampling method[38, 39], which exploits the normal distribution of bending angles dictated by the Boltzmann distribution of energy (Eq. 2). One typically generates $10^{6}-10^{7}$ chains and builds a histogram of end-to-end distances $(N[(i-1) \Delta r \leq r<i \Delta r])$ using equally spaced bins $(\Delta r)$. Normalizing this histogram by the total number of conformations yields the probability corresponding to $4 \pi r^{2} P(r) \Delta r$. Dividing this by the thin shell volume $4 \pi r^{2} \Delta r$, one can obtain the probability density function $P(r)$. The $\mathrm{J}$ factor can be read off from the y-intercept of $P(r)$ (Fig. $2 \mathrm{~A}$ ).

To compute $P(r)$ of the $\mathrm{J}$ factor of DNA shorter than about one persistence length, this 
free sampling method does not yield a statistically significant number of looped conformations in the ensemble of $10^{6}-10^{7}$ conformations. Therefore, one has to perform a biased sampling method called the umbrella sampling. In this method, one tries to restrain the two ends of the chain at close range by linking them with a stiff spring. In this method, chain conformations cannot be generated by the Gaussian sampling method because of the restraint on the end-to-end distance. Instead, one has to perform a Monte Carlo (MC) simulation where random perturbations of $\theta$ are followed by the Metropolis-Hastings acceptance criterion. This biased sampling is repeated at different end-to-end distances, and individual histograms can be combined using WHAM (Weighted Histogram Analysis Method) to obtain the probability density function[34, 28, 40]. From a set of MC simulations with different spring potentials $\left(U_{j}\right)$, a set of biased histograms centered around different end-to-end distances can be constructed. The stiffness of the spring $(K)$ and undeformed spring lengths $\left(r_{0, j}\right)$ have to be chosen carefully to cover the entire range of the end-to-end distance while allowing the histograms to be overlapped significantly. Once a full set of histograms are constructed, the unbiased probability distribution can be found using the weighted histogram analysis method (WHAM)[34, 28, 40]. Specifically, the unnormalized radial probability density in the $i$-th bin $\left(p_{i}\right)$ is given by

$$
\begin{gathered}
p_{i}=\frac{\sum_{j=1}^{N_{\text {sims }}} n_{i, j}}{\sum_{j=1}^{N_{\text {sims }}} n_{i, j} f_{j} c_{i, j}}, \\
f_{j}=\frac{1}{\sum_{i=1}^{N_{\text {bins }}} c_{i, j} p_{i}}, \quad c_{i, j}=e^{-U_{j} / k_{B} T}, \quad U_{j}=\frac{1}{2} K\left(r-r_{\circ, j}\right)^{2},
\end{gathered}
$$

where $N_{\text {sims }}$ is the number of simulations, $N_{\text {bins }}$ is the number of bins used to construct the histograms, $n_{i, j}$ is the number of conformations in the $i$-th bin $(N[(i-1) \Delta r \leq r<i \Delta r])$ of the $j$-th simulation, and $U_{j}\left(r_{i}\right)$ is the bias factor evaluated at $r_{i}$ in the $j$-th simulation. To find the optimal set of $\left\{f_{j}\right\}$, the above equations are cacluated iteratively, assuming an initial set of $\left\{f_{j}\right\}=1$, and updating the equations until $\left\{p_{i}\right\}$ converge. Finally, dividing the entire set of $\left\{p_{i}\right\}$ by $4 \pi r^{2} \Delta r$ gives $P(r)$. This calculation procedure is summarized in Table 1 using a 150-bp-long DNA chain as an example.

\section{Material and methods}

\section{1. smFRET looping assay}

The protocol for this assay is explained in great detail in our previous publication[41]. Here, we briefly highlight the key steps of the protocol. There are two different ways to construct DNA molecules for this assay. One way is to synthesize or order DNA oligos that are fluorescently labeled near the ends with donor and acceptor fluorophores. For surface immobilization, biotin also needs to be incorporated either near the end or near the center of the DNA. One should note that immobilization of DNA to the surface near its center could lead to a slightly higher looping probability[42]. Each DNA strand is designed to have 7-10 base overhang (sticky end) after hybridization. Longer DNA cannot be constructed in a 
Table 1: Step-by-step instructions with intermediate plots

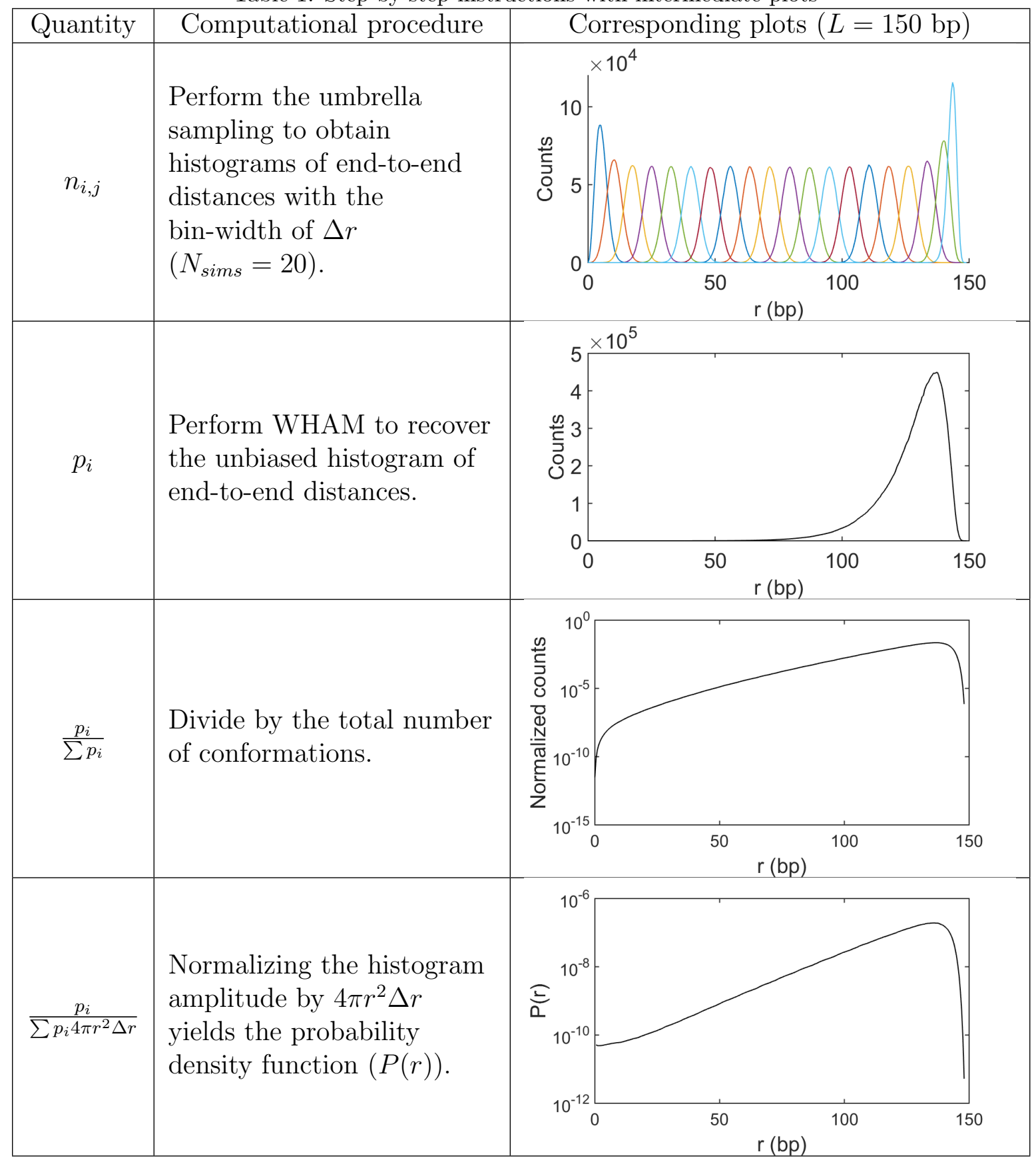


single hybridization step. Instead, one should order partially overlapping shorter oligos and ligate them into the molecule of desired length. The DNA after ligation must be denatured to eliminate unligated oligos. The successfully ligated long strands are then purified and hybridized to make intact double-stranded DNA. A more efficient and accurate method to make DNA molecules for this assay is to use PCR[41]. In this method, one orders two pairs of PCR primers that contain the required modifications (Cy3, Cy5, and biotin) and uses them to amplify the source DNA (plasmid or genomic DNA) in separate PCR reactions. The DNA products are heated and cooled, and as a result of strand exchange, DNA carrying the sticky ends (7-10 bp) and all three modifications can be unambiguously identified on the surface. The big advantage of this method over the first one is the ease of making long DNA molecules and a much lower rate of introducing sequence errors[41].

DNA molecules are then immobilized to a glass coverslip. The coverslips can be passivated with BSA, PEG, or Tween 20[43, 44]. Based on our experience, all three methods prevent nonspecific binding of DNA equally well, while only PEG and Tween-20 methods effectively prevent nonspecific binding of proteins. Among the two, we prefer PEG to Tween-20 method because the Tween-20 method involves toxic and reactive reagents. For PEGylation, we modify the glass surface with silane-PEG in a single step without going through amine modification of the surface[45]. Biotin-PEG are mixed in at 1\% (mole percentage). After NeutrAvidin incubation, DNA molecules are introduced at $100 \mathrm{pm}$. Since looping rate is low for short DNA, it is critical to use oxygen scavenging system to prolong lifetime of the dyes. We use the PCD-based oxygen scavenging system, which in our hands exhibits lower autofluorescence and slower change in $\mathrm{pH}$ compared to the glucose oxidase-based system. It is also shown that glucose oxidase-based system has high residual nuclease activity in the presence of magnesium[46].

One can perform this smFRET assay perturbatively by changing the salt concentration of the buffer (similar to stopped-flow experiments) or non-perturbatively in one salt concentration. The non-perturbative method allows measurements of both looping and unlooping rates, but may not yield good statistics for both transitions depending on DNA length and salt concentration. In this case, a salt jump experiment is preferred[26, 28].

\subsection{Single-molecule PIFE (smPIFE) binding assay}

The key idea of this assay is to place a fluorescent dye on the DNA near a protein binding site. When the protein binds to this site, it can enhance the fluorescence intensity of the adjacent dye via PIFE. To demonstrate this idea, we use the BamHI restriction enzyme, with which PIFE was thoroughly documented[47]. We constructed DNA molecules containing a single BamHI binding site (GGATCC) or two binding sites on the same DNA. For the single binding site constructs, we varied the total DNA length from $195 \mathrm{bp}$ to $2036 \mathrm{bp}$. For the double binding site constructs, we varied the separation distance between the two sites from $65 \mathrm{bp}$ to $390 \mathrm{bp}$ while keeping the total length constant. All of the tested DNA molecules were amplified and modified from pUC19 vector $(2868 \mathrm{bp}$ ) by a series of PCR reactions.

To make DNA molecules with a single BamHI site, we performed two rounds of PCR. First, we constructed DNA of various lengths with the same 5'- and 3'-end sequence by PCR amplifying the pUC19 vector. At this stage, a BamHI site is also incorporated near 
the 5' end. In the second PCR, we used two sets of primer pairs (Table 2) synthesized from IDT (Coralville, IA) to incorporate Cy3 and Biotin. These two primer pairs anneal to the common terminating sequences incorporated in the first PCR, and allow us to place the BamHI site either close to or away from the surface. Both primer pairs in Table 2 place the Cy3 dye one base pair away from the BamHI site to maximize the effect of PIFE.

To insert two BamHI binding sites in the same DNA, we first performed PCR-based sitedirected mutagenesis on the pUC19 vector to incorporate a BamHI binding site. Second, we used different pairs of PCR primers that anneal at various positions around the BamHI binding site so that the location of the BamHI site is variable in the PCR product. One of the primers also contains the second BamHI site. From this PCR, DNA constructs with a fixed length (395 bp), but various distances between the two sites are generated. These constructs were then subjected to the same PCR procedure as above for end labeling and modification.

For single-molecule PIFE (smPIFE) smPIFE experiments, we assembled a flow cell with chemically cleaned and modified glass microscope slide and coverslip[41]. Both the microscope slide and the coverslip were passivated with methoxypolyethylene glycol silane (mPEG-silane, Laysan Bio, Arab, AL) mixed with a small fraction $(\sim 1 \%)$ of biotinylated mPEG. After incubation of the surfaces with NeutrAvidin (Thermo Scientific), biotinylated DNA molecules $(\sim 40 \mathrm{pM})$ were introduced into the flow cell for surface immobilization. The imaging buffer (1050 mM Tris- $\mathrm{HCl}(\mathrm{pH} \mathrm{8}), 100 \mathrm{mM} \mathrm{NaCl}$, and $10 \mathrm{mM} \mathrm{CaCl}$ ) containing the oxygen scavenging system (5 mM PCA, $100 \mathrm{mMnM}$ PCD and $1 \mathrm{mM}$ Trolox) and BamHI was flowed into the chamber by using a programmable syringe pump (NE-1000, New Era Pump Systems). We employed an objective-type total internal reflection microscope with 532-nm laser (NT66-968, B\&W Tek, Newark, DE) to image Cy3. The fluorescence emission from Cy3 transmitted through a band pass filter was imaged onto an EMCCD (DU-897ECS0\# BV, Andor) for recording. The EMCCD (operated via an in-house software) captured images at 20 frames per second with 50-ms exposure time.

Table 2: Primer pairs. The BamHI binding site is indicated by the underlined sequence.

\begin{tabular}{|c|c|}
\hline \multicolumn{2}{|c|}{ Primer pair 1 } \\
\hline Forward & /5BioTinTEG/AAAACAAAA/iCy3/TGGATCCATAGCTGACATG \\
Backward & CACTCTGACTCGACTCG \\
\hline \multicolumn{2}{|c|}{ Primer pair 2 } \\
\hline Forward & /5Cy3/TGGATCCATAGCTGACATG \\
Backward & /5BioTinTEG/CACTCTGACTCGACTCG \\
\hline
\end{tabular}

\subsection{Data analysis}

We used lab-written MATLAB (The MathWorks, Natick, MA) codes to generate fluorescence intensity traces of $\mathrm{Cy} 3$ from the recorded movies. We identified protein bound and protein-free states from the intensity level and recorded dwell times in both states. The high 
PIFE signal was observed only when specific binding sites were present in the DNA(data not shown). Thus, we identified the low- and high- intensity states to be the protein-free and protein-bound states, respectively. We then fitted single exponential functions to the histograms of dwell times to extract the binding and unbinding rates. Then, we set an intensity threshold to distinguish the two states and collected dwell times in both states. Finally, we fitted single exponential functions to the distributions of dwell times to extract the binding and unbinding rates.

\section{Results}

\section{1. smFRET looping assay}

Although the WLC model has proven to be a robust coarse-grained model of DNA, it recently faced some challenges, especially from studies on short DNA looping. The controversy is not so much as to whether the WLC model fails or not, but rather, when and why it fails because, after all, Eq. 1 is only an approximation for small bending angles. The first in a series of studies came from the Widom group. They measured the J factor of DNA at or below 100-bp length using the ligase-dependent cyclization assay. The measured $\mathrm{J}$ factor deviated from the theoretical prediction by several orders of magnitude[23]. Some theories were proposed to explain this phenomenon, most notably, by invoking local melting[48, 49]. A year later, the same cyclization method was used to measure the looping probability at similar lengths by the Vologodskii group, but the result upheld the WLC model[24]. Recently, the DNA looping problem was investigated using single-molecule fluorescence[26, 27]. Especially, the Ha group performed the J factor measurement with short DNA and extracted $\mathrm{J}$ factor values seemingly much higher than the WLC model prediction. This study reignited interest of the field on this topic. However, technical concerns about the extraction of the $\mathrm{J}$ factor[50] and salt effects[51] remain.

The measurables in the smFRET assay (Fig. 3A) are the looping rate $\left(k_{\text {loop }}\right)$, the unlooping rate $\left(k_{\text {unloop }}\right)$, or the ratio of the two $\left(k_{\text {loop }} / k_{\text {unloop }}\right)$. In the smFRET assay, looping and unlooping of DNA lead to fluorescence intensity fluctuations. High $\mathrm{Cy} 3$ and low $\mathrm{Cy} 5$ signals correspond to the unlooped state whereas low $\mathrm{Cy} 3$ and high $\mathrm{Cy} 5$ signals correspond to the looped state (Fig. 3B). From the intensity fluctuations, individual dwell times in the looped state $\left(\tau_{\text {loop }}\right)$ and the unlooped state $\left(\tau_{\text {unloop }}\right)$ are recorded and averaged to obtain the mean dwell times or lifetimes $\left(\left\langle\tau_{\text {loop }}\right\rangle\right.$ and $\left.\left\langle\tau_{\text {unloop }}\right\rangle\right)$. The looping and unlooping rates are equal to the inverses of these lifetimes: $k_{\text {loop }}=\left\langle\tau_{\text {unloop }}\right\rangle^{-1}$ and $k_{\text {unloop }}=\left\langle\tau_{\text {loop }}\right\rangle^{-1}$. These apparent rates, however, depend on the affinity between the sticky ends. Therefore, to extract quantities such as the $\mathrm{J}$ factor attributable to DNA conformation only, the looping and unlooping rates must be normalized against the rates for association $\left(k_{o n}\right)$ and dissociation $\left(k_{\text {off }}\right)$ between the sticky ends. These rates are measured in a similar FRET experiment using the truncated end segments of the DNA molecule (inner panel, Fig. 3A).

In this section, we carefully examine the relationship between these measurables and the $\mathrm{J}$ factor and contend that the $\mathrm{J}$ factor cannot be unambiguously determined from these measurables. Therefore, although the apparent rates provide valuable insights into DNA bendability, their relationship to the $\mathrm{J}$ factor must be interpreted with caution. 
(A)
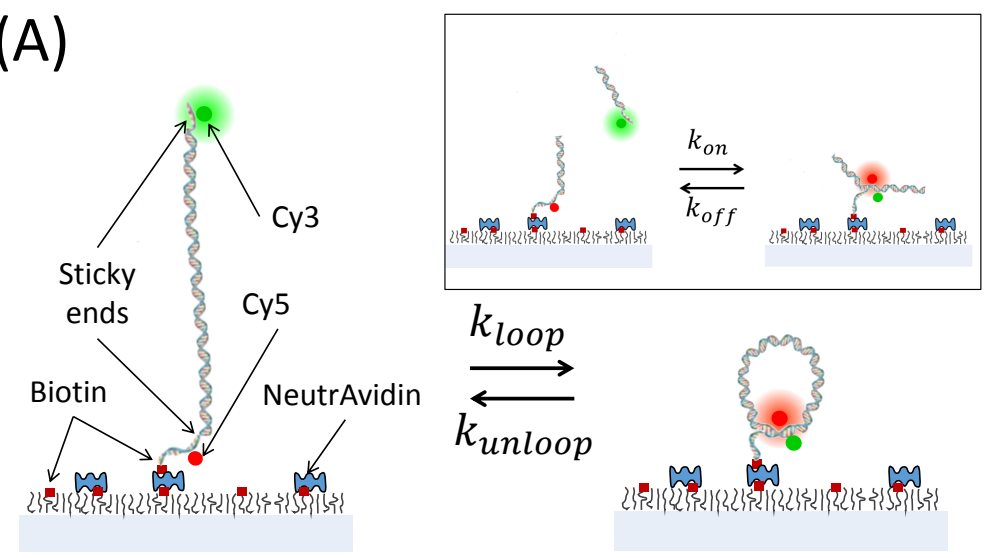

(B)

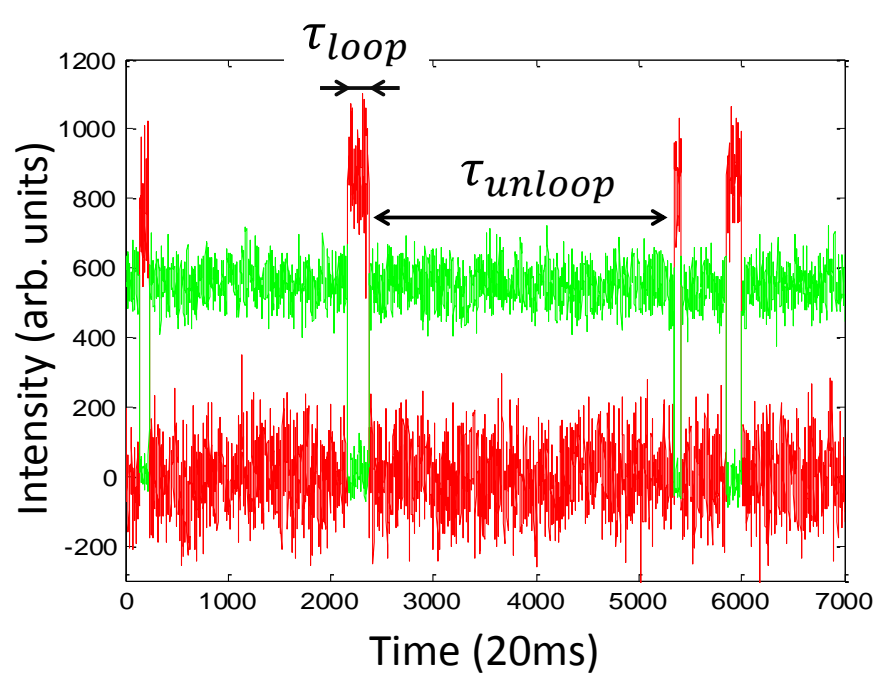

Figure 3: Single-molecule FRET looping assay. (A) The experimental setup. Double-stranded DNA molecules with complementary overhangs (sticky ends) and the FRET pair (Cy3 and Cy5) are immobilized on the glass coverslip, and fluorescence signals are observed by an objective-type total internal microscope. DNA molecules can be trapped in the looped state due to base pairing between the sticky ends. They loop and unloop with apparent rates of $k_{\text {loop }}$ and $k_{\text {unloop }}$, respectively. The boxed inset shows the single-molecule measurement of the association $\left(k_{o n}\right)$ and dissociation $\left(k_{o f f}\right)$ rates between the unlinked sticky ends. (B) Time trajectories of $\mathrm{Cy} 3$ and $\mathrm{Cy} 5$ fluorescence intensities. The intensities fluctuate between two levels due to reversible looping and unlooping. The FRET efficiency is low in the unlooped state and high in the looped state. Individual dwell times in the looped $\left(\tau_{\text {loop }}\right)$ and unlooped $\left(\tau_{\text {unloop }}\right)$ states are used to build dwell time histograms, from which the unlooping rate $\left(k_{\text {unloop }}\right)$ and the looping rate $\left(k_{\text {loop }}\right)$ can be determined.

\subsubsection{Looping rate}

First, let's consider a normal bimolecular reaction between two complementary singlestranded DNA molecules. Without losing generality, we consider one to be fixed in space and the other one freely diffusing about. Association (annealing) between them can be 
schematized by a pseudo first order reaction:

$$
\mathrm{A} \underset{k_{o f f}}{\stackrel{k_{o n}}{\rightleftarrows}} \mathrm{AB}
$$

where $\mathrm{A}$ is the single-stranded state of $\mathrm{A}$, and $\mathrm{AB}$ is the duplex state where base pairs are formed with $\mathrm{B}$. The association rate of $\mathrm{B}$ to $\mathrm{A}\left(k_{o n}\right)$ is proportional to the concentration of $\mathrm{B}([B])$, and therefore

$$
k_{\text {on }}=k_{\text {on }}^{\prime}[B] .
$$

The second-order rate constant $k_{o n}^{\prime}$ can be measured from the concentration dependence of $k_{o n}$. The dissociation rate of $\mathrm{B}$ from $\mathrm{A}\left(k_{o f f}\right)$ is concentration independent.

In the smFRET assay, the high FRET state is stabilized by base pairing between the sticky ends of a DNA molecule. Association between two ends of the same molecule can be similarly described using a first-order reaction

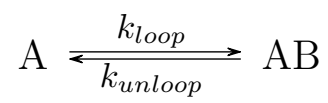

where $k_{\text {loop }}$ and $k_{\text {unloop }}$ are the first-order rates for low-to-high and high-to-low FRET transitions, respectively. $k_{\text {loop }}$ should be proportional to the effective concentration of one end of the DNA molecule in close proximity to the other end, which is the $\mathrm{J}$ factor $(J)$. Hence, we can simply use $J$ as the concentration in Eq. 10 to relate $J$ to the measured $k_{\text {loop }}$,

$$
k_{\text {loop }} \approx k_{\text {on }}^{\prime} J \quad \text { or } \quad J \approx \frac{k_{\text {loop }}}{k_{\text {on }}^{\prime}}
$$

Therefore by dividing the first-order rate of loop formation $\left(k_{\text {loop }}\right)$ by the second-order rate constant of duplex formation between the sticky ends $\left(k_{o n}^{\prime}\right)$, one can extract the $\mathrm{J}$ factor in molar units. This is the expression used in our previous study[27]. In comparison, Vafabakhsh and Ha divided $k_{\text {loop }}+k_{\text {unloop }}$ by $k_{\text {on }}^{\prime}$ to extract the $\mathrm{J}$ factor in their study[26]. This expression, however, is expected to overestimate the $\mathrm{J}$ factor as $k_{\text {unloop }}$ increases as DNA loop becomes shorter (explained in Eq. 13 and Fig. 4A). Implicit in Eq. 12 is the assumption that $k_{\text {on }}^{\prime}$ is not affected by the presence of the loop.

\subsubsection{Unlooping rate}

Experimentally, looping of DNA shorter than one persistence length is extremely rare, which motivates experimenters to use extreme conditions (high salt, high ligase concentration). We find that a more robust way to test the WLC model in standard salt conditions is to measure the unlooping rate of a small DNA loop. In this assay, DNA molecules with sticky ends are first prepared in the looped state by using a high salt buffer, which are then induced to break open by switching to a low salt buffer. From the exponential decay of the looped population, the unlooping rate $\left(k_{\text {unloop }}\right)$ or the looped-state lifetime $\left(\left\langle\tau_{\text {loop }}\right\rangle\right)$ can be extracted. This loop lifetime decreases as the loop size becomes smaller because of higher bending stress.

This relationship can be understood based on the transition state theory. Imagine the transition state of duplex dissociation is $\Delta r$ away from the ground state at $r_{0} . r_{0}$ is the length 
of the duplex between the sticky ends in the ground state. According to the transition state theory, the dissociation rate is proportional to the probability of occupying the transition state. The shear force by the loop lowers the free energy of the transition state more than that of the ground state by $\Delta A$ (Fig. 4 ). Therefore, $k_{\text {unloop }}$, which is the dissociation rate of the duplex holding the loop, would be faster than that without the loop $\left(k_{\text {off }}\right.$, Eq. 9$)$ by a Boltzmann factor:

$$
k_{\text {unloop }}=k_{\text {off }} \exp \left(\Delta A / k_{B} T\right) .
$$

Using a linear approximation, the free energy difference can be expressed in terms of the shear force $(f)$ exerted on the duplex formed between the sticky ends of the loop (Fig. 4A):

$$
k_{\text {unloop }}=k_{\text {off }} \exp \left(f \Delta r / k_{B} T\right)
$$

which is equivalent to the well-known Bell model of force-induced bond breakage[52]. $f$ can be calculated as a function of loop size $L$ by differentiating the free energy by endto-end distance. But this requires knowing the functional form of $P(r)$ at small $r$, which is complicated even in approximate forms[34]. Instead, one can run an umbrella sampling simulation with a restraining potential around $r_{0}[28]$. The force can then be easily computed from the variance of the end-to-end distances[53]. Using this unlooping assay, we previously showed that the linear relationship between the logarithm of the loop lifetime and $f$ breaks down at the loop size of $60 \mathrm{bp}$ with sodium only and $100 \mathrm{bp}$ with $5 \mathrm{mM}$ magnesium[28]. The mechanism behind this critical limit still needs to be investigated. Based on previous experimental[54, 51] and computational[55, 56, 57] studies, it likely reflects the kinking transition of a dinucleotide. Fraying around preexisting nicks could also relax the bending stress $[58,59]$, but such transition seems to be preceded by kinking[60].

\subsubsection{Equilibrium fraction}

Another method to extract the $\mathrm{J}$ factor experimentally is to measure the equilibrium fraction of the looped state. This method has been used in the analysis of tethered particle motion experiments $[61,62,63]$, and appears to be applicable to smFRET experiments that record looping and unlooping in a reversible manner[27]. From single-molecule trajectories of looping and unlooping, one can calculate the ratio of the looped time $\left(\tau_{\text {loop }}\right)$ to the unlooped

time $\left(\tau_{\text {unloop }}\right)$. Separately, one can measure the affinity between the molecules that stabilize the DNA loop (protein-protein, protein-dsDNA, or ssDNA-ssDNA interaction) $\left(K_{D}\right)$, similar to Eq. 9. The equilibrium constant $K_{D}$ in molar units is measured by dividing the first order dissociation rate constant $\left(k_{o f f}\right)$ by the second order association constant $\left(k_{o n}^{\prime}\right)$. Using a simple thermodynamic argument with the assumption that $K_{D}$ is the same in the context of the loop, the $\mathrm{J}$ factor can be extracted from three measurables as

$$
J \approx K_{D} \frac{\tau_{\text {loop }}}{\tau_{\text {unloop }}}
$$

Expressing this in terms of rates, we obtain

$$
J=\frac{k_{\text {off }}}{k_{\text {on }}^{\prime}} \frac{k_{\text {loop }}}{k_{\text {unloop }}}<\frac{k_{\text {loop }}}{k_{\text {on }}^{\prime}}
$$


(A)

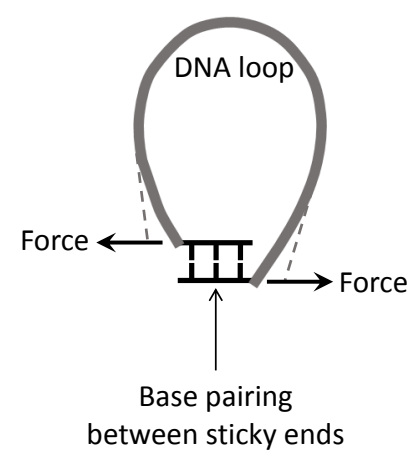

(B)

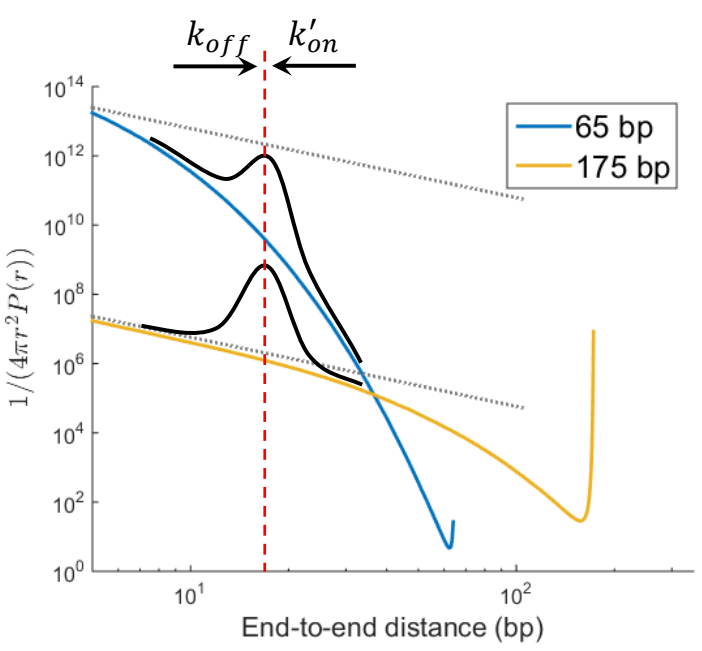

Figure 4: The influence of a tightly bent DNA on base pairing between sticky ends. (A) Schematic of a DNA loop stabilized by end-to-end annealing. The duplex formed between the sticky ends experiences a shear force due to the elasticity of the DNA loop. (B) The free energy landscape of duplex association and dissociation. The blue (65 bp) and yellow (175 bp) curves are the inverse probability density functions used in Eq. 5, which are calculated from the simulation as described in 2.2. On a log y-axis, the inverse probability density function can be interpreted as the free energy landscape (Eq. 5). The $\mathrm{x}$-axis is also scaled logarithmically to highlight the slope of the landscape. The black curves represent the transition barrier that the end-to-end distance must cross for duplex formation or dissociation. The red dashed line indicates the transition state position. Short DNA (blue) has a heavily tilted landscape which increases duplex dissociation rate $\left(k_{o f f}\right)$ and decreases duplex association rate $\left(k_{o n}^{\prime}\right)$. For reference, the landscapes corresponding to the association of unlinked sticky ends is shown as gray dotted lines.

where the latter inequality holds because $k_{\text {unloop }}>k_{\text {off }}$ according to Eq. 13. Therefore, the $\mathrm{J}$ factor extracted this way is smaller than the $\mathrm{J}$ factor extracted from Eq. 12. The discrepancy between the two expressions will grow larger as the DNA loop becomes smaller.

The takeaway message is that the dependence of the $\mathrm{J}$ factor on measurable rates and equilibrium constants is more complicated than generally assumed. The $\mathrm{J}$ factor was originally formulated for long DNA[21, 20], where the effect of the loop on $k_{o n}^{\prime}, k_{o f f}$, and $K_{D}$ would be negligible. The effect of the loop on base pairing equilibrium can be visualized by the free energy landscape. In Fig. 4, the free energy landscape of the end-to-end distance $r$ of DNA is plotted for 65-bp (blue) and 175-bp (yellow) DNA. For comparison, the free energy landscapes from contant $P(r)$ 's are plotted as gray dotted lines, which follow a power law with exponent equal to -2 . Overlaid in black are the transition barriers for duplex formation. For long DNA (175 bp), the free energy landscape at small $r$ has a similar slope to the gray line, which indicates that base pairing equilibrium between sticky ends would not be greatly affected by the loop. In contrast, the free energy landscape for the short DNA(65 bp) is heavily tilted compared to the gray line, which lowers the transition barrier for $k_{o f f}$ and raises it for $k_{o n}^{\prime}$. Therefore, the assumption of loop-independent $k_{o n}^{\prime}$ used in Eq. 12 or loop-independent $K_{D}$ used in Eq. 15 is no longer valid for short dsDNA.

A more theoretically correct treatment of the $\mathrm{J}$ factor is given only recently by Mulligan 
et al.[64, 65] by solving the Fokker-Planck equation using the minimum free energy path. In this model, both the looping and unlooping rates scale with the J factor. Especially, the looping rate $\left(k_{\text {loop }}\right)$ scales with the $\mathrm{J}$ factor with an exponent less than one, whose values depends on the reaction distance and geometry.

\section{2. smPIFE protein binding assay}

\subsubsection{Demonstration of PIFE}

Intersegmental transfer of proteins between different dsDNA molecules has been extensively studied in solution $[66,67]$. In these studies, a fluorescent dye is covalently linked to DNA near a protein binding site so that protein binding causes detectable change in fluorescence intensity of the dye. Based on this photophysical phenomenon, the rate of protein-DNA association was measured as a function of competitor DNA concentration in stopped flow experiments. Following a similar approach, we use a well-documented fluorescence technique called protein-induced fluorescence enhancement (PIFE)[47] to investigate intersegmental transfer within the same DNA molecule. To unambiguously study transfer between segments of the same DNA, we monitored the fluorescence change from surfaceimmobilized DNA molecules.

(A)

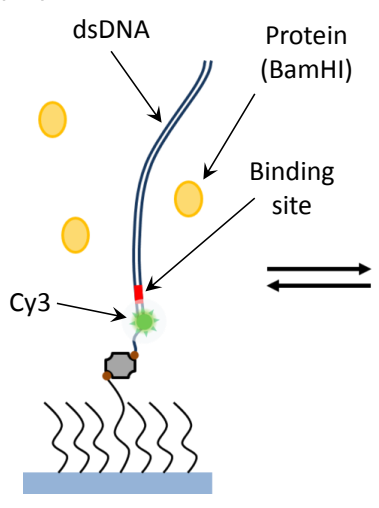

(B)

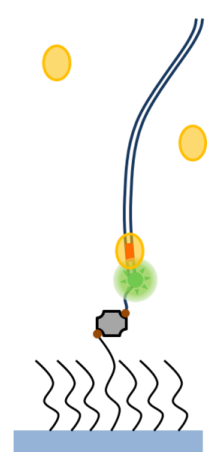

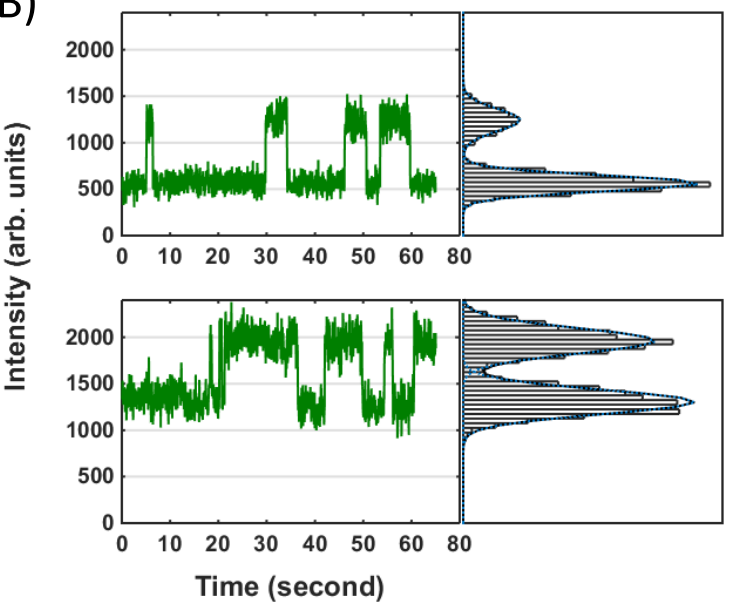

Figure 5: Demonstration of PIFE. (A) Experimental setup. DNA molecules with Cy3 near a single BamHI binding site are immobilized on the glass coverslip. BamHI in the solution can reversibly bind and unbind the site, which leads to fluctuations in the Cy3 fluorescence intensity. (B) Example time trajectories of Cy3 fluorescence intensity and corresponding intensity distributions of the two different fluorophore configurations. The top trace is obtained from a DNA molecule carrying the binding site and terminally linked Cy3 away from the surface, and the bottom trace from a DNA molecule with the binding site and backbonelinked $\mathrm{Cy} 3$ close to the surface. The fluorescence intensity in the top trace is lower than that in the bottom because of the decaying intensity profile of the evanescent excitation of the TIR microscope. The bone-linked $\mathrm{Cy} 3$ in the bottom trace exhibits a lower intensity The lower intensity fold-change seen from the backbone-linked Cy3 compared to the terminally linked Cy3 is probably due to the restricted cis-trans isomerization of the backbone-linked $\mathrm{Cy} 3$.

We used BamHI restriction enzyme as a model system because it is commercially available 
and is shown to exhibit dramatic PIFE effect[47]. PIFE is most effective when the BamHI binding site is only one base pair away from a fluorescent dye. We prepared Cy3-labeled DNA fragments with a BamHI binding site located one base pair away from the Cy3 dye. We observed the fluorescence intensity of $\mathrm{Cy} 3$ from DNA molecules jump to a higher level only when the imaging buffer contained BamHI proteins. We also showed that the rate of intensity change from low to high increased linearly with BamHI concentration while the rate of change from high to low did not. Fig. 5(B) shows typical fluorescence traces of single $\mathrm{Cy} 3$, which clearly show intensity fluctuations. The top trace is obtained from DNA molecules carrying the BamHI site and Cy3 near the solution-end of the DNA. In this construct, Cy3 was linked to the 5'-end. In comparison, the bottom trace is obtained from a different DNA construct with the BamHI site and Cy3 near the surface-attached end. Because the 5'-end was used for biotinylation, Cy3 was internally linked through the DNA backbone. Interestingly, the PIFE signal (fold-change in intensity) was $\sim 2.5$ for the 5 '-end linked Cy3, but less than 2 for the backbone-linked Cy3 (Fig. 5(B)). We speculate that this is because cis-trans isomerization of $\mathrm{Cy} 3$, which is thought to be responsible for PIFE, is more restricted for the backbone-linked Cy3, thus a lower PIFE signal.

\subsubsection{The effect of DNA length on target search kinetics of BamHI}

Having demonstrated PIFE with our BamHI-DNA system, we next set out to investigate the role of nonspecific binding in target search mechanism. As shown for other proteins[66], BamHI may bind DNA in a nonspecific manner and employ one-dimensional sliding to search its target on DNA. We measured the binding $\left(k_{o n}\right)$ and unbinding $\left(k_{o f f}\right)$ rates of BamHI using DNA molecules containing a single binding site ranging in length from $200 \mathrm{bp}$ to 2000 bp. We used $25 \mathrm{~nm}$ of BamHI and added 10\% (v/v) DMSO to increase the binding affinity of the enzyme. Fig. 6(A) shows the binding and unbinding rates as a function of DNA length. The binding rate increases in a length-dependent manner up to $\sim 700 \mathrm{bp}$ and reaches a plateau beyond that length. In contrast, the unbinding rate stays constant, independent of the DNA length. A similar length-dependent target search kinetics has been observed in previous studies $[67,66,68,69]$, and is attributable to one-dimensional sliding initiated by nonspecific landing of the protein on DNA.

\subsection{The effect of a second site on target search kinetics of BamHI}

Having established the baseline effect of nonspecific binding on target search kinetics, we next asked whether a second BamHI site on the same DNA as the first site could shorten target recognition time through intersegmental transfer. A protein partially associated with DNA can be exchanged with another competing DNA or protein in a concentration dependent manner. Thus, the apparent dissociation rate $\left(k_{o f f}\right)$ of a DNA-bound protein is given by

$$
k_{o f f}=k_{o f f, 0}+k_{e x c h} c,
$$

where $k_{o f f, 0}$ is the unperturbed dissociation rate, $k_{\text {exch }}$ is the concentration-dependent firstorder exchange rate, $c$ is the concentration of the competitor. This competitor effect can also be presented by a second site on the same DNA through looping. The transfer rate would 
then depend on the effective concentration of the invading site near the occupied site, which is equivalent to the $\mathrm{J}$ factor. Since the $\mathrm{J}$ factor of DNA is peaked at $10^{-7} \mathrm{M}$ near $500 \mathrm{bp}$, the rate of intersegmental transfer is expected to be highest around $500 \mathrm{bp}$. We note that contact equilibrium between two sites in the interior of a WLC (interior looping) is different from that between the ends (end-to-end looping), but difference is only marginal[36]. As an example, substituting $J=10^{-7} \mathrm{M}$ into $c$ in Eq. 17 and using the rates measured for the Escherichia coli nucleoid-associated protein Fis[70], we can estimate the dissociation rate of Fis to increase by a factor of 7 due to DNA looping.

(A)

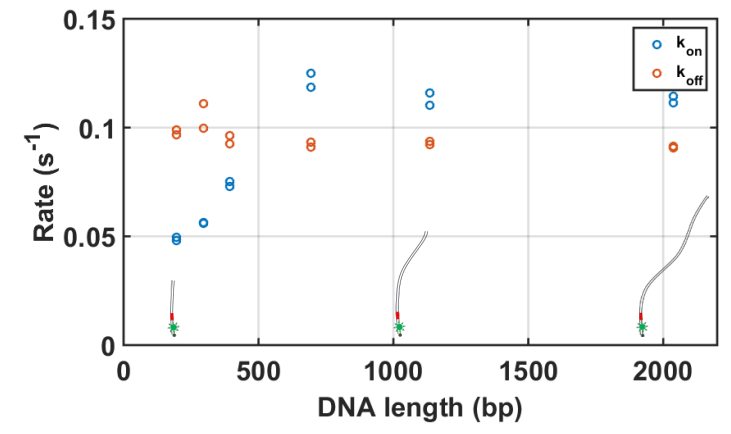

(B)

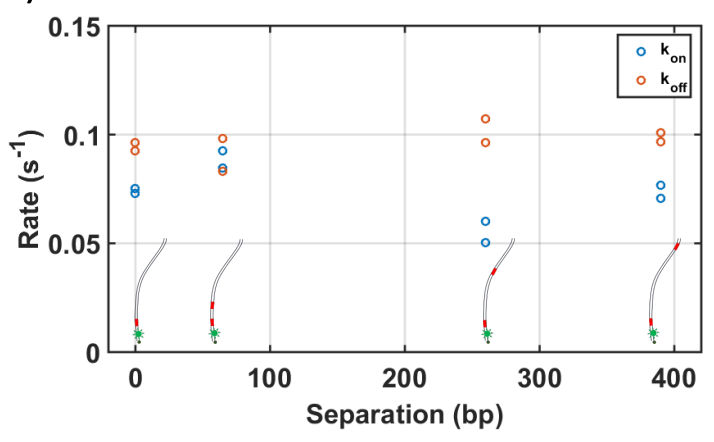

Figure 6: The binding $\left(k_{o n}\right)$ and unbinding $\left(k_{o f f}\right)$ rates of BamHI. (A) Results from DNA constructs with a single binding site. Cartoon depictions of corresponding DNA constructs are shown below the data points. The BamHI site (red) and Cy3 (green) are located near the surface-facing end of the DNA. (B) Results from DNA constructs with two distal binding sites. Cartoon depictions of corresponding DNA constructs are shown below the data points. The position of the Cy3-labeled BamHI site is fixed while the second site is placed at increasing distances from it.

We prepared 395-bp long DNA molecules carrying two BamHI sites separated by various distances $\left(65 \mathrm{bp}, 260 \mathrm{bp}\right.$, and $390 \mathrm{bp}$ ). The binding $\left(k_{o n}\right)$ and unbinding $\left(k_{o f f}\right)$ rates to and from the Cy3-neighboring site were measured, and the results are shown in Fig. 6(B) along with the rates measured from the control sequence with a single binding site. We kept the concentration of BamHI low $(\sim 25 \mathrm{nM})$ in order to prevent saturation of all the available BamHI binding sites. As the separation distance increases toward $\sim 500$ bp where the $\mathrm{J}$ factor is maximum, the contact probability between the two distal sites is predicted to increase dramatically as shown in Fig. 2B. Consequently, BamHI bound at one site would have a higher chance to hop over to the other site with increasing separation distance, and therefore, we expected to see a corresponding increase in both $k_{o n}$ and $k_{o f f}$. However, Fig. 6(B) shows no significant change in either rate compared to the control construct. Although in need of more supporting data, our preliminary result shows that BamHI does not employ intersegmental transfer as a major target search mechanism.

\section{Conclusions}

In conclusion, we introduce single-molecule fluorescence assays to study biophysics of DNA loops and DNA looping mediated intersegmental transfer. The enzyme-free smFRET 
looping assay overcomes many of the technical challenges presented by the ligase-dependent cyclization assay. Since the smFRET assay can also be performed in a reversible manner, one can focus on the unlooping transition, which is well suited to studying the energetics of strong DNA bending. Although this smFRET assay and other looping assays produce rates that give us insights into elastic properties of DNA, we argue that extraction of the J factor from these rates is highly problematic, especially for small DNA loops where the loop stabilizing interaction is under high shear force. We also introduce a novel experimental platform based on smPIFE to investigate DNA looping-mediated intersegmental transfer. We demonstrated the feasibility of this assay using the BamHI restriction enzyme. Since PIFE is a robust phenomenon, we anticipate that our smPIFE assay can be applied to a wide array of DNA binding proteins to delineate their target search mechanisms.

\section{Acknowledgements}

The authors thank the Harold Kim lab members for useful discussions. This work was supported by National Institutes of Health (R01GM112882) and National Science Foundation (1517507).

\section{References}

[1] W. K. Olson, V. B. Zhurkin, Modeling dna deformations, Current opinion in structural biology 10 (3) (2000) 286-297.

[2] J. G. Elias, D. Eden, Transient electric birefringence study of the persistence length and electrical polarizability of restriction fragments of dna, Macromolecules 14 (2) (1981) 410-419.

[3] J. Bednar, P. Furrer, V. Katritch, A. Stasiak, J. Dubochet, A. Stasiak, Determination of dna persistence length by cryo-electron microscopy. separation of the static and dynamic contributions to the apparent persistence length of dna, Journal of molecular biology 254 (4) (1995) 579-594.

[4] S. B. Smith, Y. Cui, C. Bustamante, Overstretching b-dna: the elastic response of individual doublestranded and single-stranded dna molecules, Science 271 (5250) (1996) 795-799.

[5] C. Bouchiat, M. Wang, J.-F. Allemand, T. Strick, S. Block, V. Croquette, Estimating the persistence length of a worm-like chain molecule from force-extension measurements, Biophysical journal 76 (1) (1999) 409-413.

[6] S. D. Levene, S. M. Giovan, A. Hanke, M. J. Shoura, The thermodynamics of dna loop formation, from $\mathrm{j}$ to z, Biochem. Soc. Trans 41 (2013) 513-518.

[7] H. G. Garcia, P. Grayson, L. Han, M. Inamdar, J. Kondev, P. C. Nelson, R. Phillips, J. Widom, P. A. Wiggins, Biological consequences of tightly bent dna: the other life of a macromolecular celebrity, Biopolymers 85 (2) (2007) 115-130.

[8] S. Kadauke, G. A. Blobel, Chromatin loops in gene regulation, Biochimica et Biophysica Acta (BBA)Gene Regulatory Mechanisms 1789 (1) (2009) 17-25.

[9] L. M. Bond, J. P. Peters, N. A. Becker, J. D. Kahn, L. J. Maher, Gene repression by minimal lac loops in vivo, Nucleic acids research 38 (22) (2010) 8072-8082.

[10] A. N. Yadon, B. N. Singh, M. Hampsey, T. Tsukiyama, Dna looping facilitates targeting of a chromatin remodeling enzyme, Molecular cell 50 (1) (2013) 93-103.

[11] A. J. Andrews, K. Luger, Nucleosome structure (s) and stability: variations on a theme, Annual review of biophysics 40 (2011) 99-117.

[12] T. Baker, N. Olson, S. Fuller, Adding the third dimension to virus life cycles: three-dimensional reconstruction of icosahedral viruses from cryo-electron micrographs, Microbiology and Molecular Biology Reviews 63 (4) (1999) 862-922. 
[13] Z. Dai, Y. Xiong, X. Dai, The pattern and evolution of looped gene bendability, Molecular biology and evolution (2013) mst188.

[14] A. L. Hughes, O. J. Rando, Mechanisms underlying nucleosome positioning in vivo, Annual review of biophysics 43 (2014) 41-63.

[15] T. Ruusala, D. M. Crothers, Sliding and intermolecular transfer of the lac repressor: kinetic perturbation of a reaction intermediate by a distant dna sequence, Proceedings of the National Academy of Sciences 89 (11) (1992) 4903-4907.

[16] C. Q. Pan, S. E. Finkel, S. E. Cramton, J.-A. Feng, D. S. Sigman, R. C. Johnson, Variable structures of fis-dna complexes determined by flanking dna-protein contacts, Journal of molecular biology 264 (4) (1996) 675-695.

[17] B. A. Lieberman, S. K. Nordeen, Dna intersegment transfer, how steroid receptors search for a target site, Journal of Biological Chemistry 272 (2) (1997) 1061-1068.

[18] D. Skoko, B. Wong, R. C. Johnson, J. F. Marko, Micromechanical analysis of the binding of dna-bending proteins hmgb1, nhp6a, and hu reveals their ability to form highly stable dna-protein complexes, Biochemistry 43 (43) (2004) 13867-13874.

[19] M. Doucleff, G. M. Clore, Global jumping and domain-specific intersegment transfer between dna cognate sites of the multidomain transcription factor oct-1, Proceedings of the National Academy of Sciences 105 (37) (2008) 13871-13876.

[20] D. Shore, J. Langowski, R. L. Baldwin, Dna flexibility studied by covalent closure of short fragments into circles, Proceedings of the National Academy of Sciences 78 (8) (1981) 4833-4837.

[21] H. Jacobson, W. H. Stockmayer, Intramolecular reaction in polycondensations. i. the theory of linear systems, The Journal of Chemical Physics 18 (12) (1950) 1600-1606.

[22] J. P. Peters, L. J. Maher, Dna curvature and flexibility in vitro and in vivo, Quarterly reviews of biophysics 43 (01) (2010) 23-63.

[23] T. E. Cloutier, J. Widom, Spontaneous sharp bending of double-stranded dna, Molecular cell 14 (3) (2004) 355-362.

[24] Q. Du, C. Smith, N. Shiffeldrim, M. Vologodskaia, A. Vologodskii, Cyclization of short dna fragments and bending fluctuations of the double helix, Proceedings of the National Academy of Sciences of the United States of America 102 (15) (2005) 5397-5402.

[25] K. A. Schallhorn, K. O. Freedman, J. M. Moore, J. Lin, P. C. Ke, Single-molecule dna flexibility in the presence of base-pair mismatch, Applied Physics Letters 87 (3) (2005) 033901.

[26] R. Vafabakhsh, T. Ha, Extreme bendability of dna less than 100 base pairs long revealed by singlemolecule cyclization, Science 337 (6098) (2012) 1097-1101.

[27] T. T. Le, H. D. Kim, Measuring shape-dependent looping probability of dna, Biophysical journal 104 (9) (2013) 2068-2076.

[28] T. T. Le, H. D. Kim, Probing the elastic limit of dna bending, Nucleic acids research 42 (16) (2014) 10786-10794.

[29] A. J. Pollak, A. T. Chin, F. L. Brown, N. O. Reich, Dna looping provides for intersegmental hopping by proteins: a mechanism for long-range site localization, Journal of molecular biology 426 (21) (2014) 3539-3552.

[30] A. J. Pollak, A. T. Chin, N. O. Reich, Distinct facilitated diffusion mechanisms by e. coli type ii restriction endonucleases, Biochemistry 53 (45) (2014) 7028-7037.

[31] K. S. Lee, A. B. Marciel, A. G. Kozlov, C. M. Schroeder, T. M. Lohman, T. Ha, Ultrafast redistribution of e. coli ssb along long single-stranded dna via intersegment transfer, Journal of molecular biology 426 (13) (2014) 2413-2421.

[32] A. Brunet, C. Tardin, L. Salomé, P. Rousseau, N. Destainville, M. Manghi, Dependence of dna persistence length on ionic strength of solutions with monovalent and divalent salts: A joint theoryexperiment study, Macromolecules.

[33] J. F. Marko, Biophysics of protein-dna interactions and chromosome organization, Physica A: Statistical Mechanics and its Applications 418 (2015) 126-153.

[34] N. Becker, A. Rosa, R. Everaers, The radial distribution function of worm-like chains, The European 
Physical Journal E 32 (1) (2010) 53-69.

[35] J. Shimada, H. Yamakawa, Ring-closure probabilities for twisted wormlike chains. application to dna, Macromolecules 17 (4) (1984) 689-698.

[36] C. Hyeon, D. Thirumalai, Kinetics of interior loop formation in semiflexible chains, The Journal of chemical physics 124 (10) (2006) 104905.

[37] J.-F. Allemand, S. Cocco, N. Douarche, G. Lia, Loops in dna: an overview of experimental and theoretical approaches, The European Physical Journal E 19 (3) (2006) 293-302.

[38] L. Czapla, D. Swigon, W. K. Olson, Sequence-dependent effects in the cyclization of short dna, Journal of Chemical Theory and Computation 2 (3) (2006) 685-695.

[39] N. J. Agrawal, R. Radhakrishnan, P. K. Purohit, Geometry of mediating protein affects the probability of loop formation in dna, Biophysical journal 94 (8) (2008) 3150-3158.

[40] H. Salari, B. Eslami-Mossallam, M. Naderi, M. Ejtehadi, Extreme bendability of dna double helix due to bending asymmetry, arXiv preprint arXiv:1505.03138.

[41] T. T. Le, H. D. Kim, Studying dna looping by single-molecule fret, JoVE (Journal of Visualized Experiments) (88) (2014) e51667-e51667.

[42] J. T. Waters, H. D. Kim, Equilibrium statistics of a surface-pinned semiflexible polymer, Macromolecules 46 (16) (2013) 6659-6666.

[43] B. Hua, K. Y. Han, R. Zhou, H. Kim, X. Shi, S. C. Abeysirigunawardena, A. Jain, D. Singh, V. Aggarwal, S. A. Woodson, et al., An improved surface passivation method for single-molecule studies, nature methods 11 (12) (2014) 1233-1236.

[44] H. Pan, Y. Xia, M. Qin, Y. Cao, W. Wang, A simple procedure to improve the surface passivation for single molecule fluorescence studies, Physical biology 12 (4) (2015) 045006.

[45] A. Gust, A. Zander, A. Gietl, P. Holzmeister, S. Schulz, B. Lalkens, P. Tinnefeld, D. Grohmann, A starting point for fluorescence-based single-molecule measurements in biomolecular research, Molecules 19 (10) (2014) 15824-15865.

[46] G. Senavirathne, J. Liu, M. A. Lopez Jr, J. Hanne, J. Martin-Lopez, J.-B. Lee, K. E. Yoder, R. Fishel, Widespread nuclease contamination in commonly used oxygen-scavenging systems, Nature methods 12 (10) (2015) 901-902.

[47] H. Hwang, H. Kim, S. Myong, Protein induced fluorescence enhancement as a single molecule assay with short distance sensitivity, Proceedings of the National Academy of Sciences 108 (18) (2011) 7414-7418.

[48] J. Yan, J. F. Marko, Localized single-stranded bubble mechanism for cyclization of short double helix dna, Physical review letters 93 (10) (2004) 108108.

[49] P. Ranjith, P. S. Kumar, G. I. Menon, Distribution functions, loop formation probabilities, and forceextension relations in a model for short double-stranded dna molecules, Physical review letters 94 (13) (2005) 138102.

[50] A. Vologodskii, M. D. Frank-Kamenetskii, Strong bending of the dna double helix, Nucleic acids research 41 (14) (2013) 6785-6792.

[51] A. P. Fields, E. A. Meyer, A. E. Cohen, Euler buckling and nonlinear kinking of double-stranded dna, Nucleic acids research 41 (21) (2013) 9881-9890.

[52] G. I. Bell, Models for the specific adhesion of cells to cells, Science 200 (4342) (1978) 618-627.

[53] W. Hwang, Calculation of conformation-dependent biomolecular forces, The Journal of chemical physics 127 (17) (2007) 175104.

[54] Q. Du, A. Kotlyar, A. Vologodskii, Kinking the double helix by bending deformation, Nucleic acids research 36 (4) (2008) 1120-1128.

[55] F. Lankaš, R. Lavery, J. H. Maddocks, Kinking occurs during molecular dynamics simulations of small dna minicircles, Structure 14 (10) (2006) 1527-1534.

[56] J. Mitchell, C. Laughton, S. A. Harris, Atomistic simulations reveal bubbles, kinks and wrinkles in supercoiled dna, Nucleic acids research (2011) gkq1312.

[57] R. N. Irobalieva, J. M. Fogg, D. J. Catanese, T. Sutthibutpong, M. Chen, A. K. Barker, S. J. Ludtke, S. A. Harris, M. F. Schmid, W. Chiu, et al., Structural diversity of supercoiled dna, Nature communications 6 . 
[58] C. Kim, O.-c. Lee, J.-Y. Kim, W. Sung, N. K. Lee, et al., Dynamic release of bending stress in short dsdna by formation of a kink and forks, Angewandte Chemie International Edition 54 (31) (2015) 8943-8947.

[59] P. Cong, L. Dai, H. Chen, J. R. van der Maarel, P. S. Doyle, J. Yan, Revisit the anomalous bending elasticity of sharply bent dna, arXiv preprint arXiv:1507.06354.

[60] R. M. Harrison, F. Romano, T. E. Ouldridge, A. A. Louis, J. P. Doye, Coarse-grained modelling of strong dna bending ii: Cyclization, arXiv preprint arXiv:1506.09008.

[61] L. Han, H. G. Garcia, S. Blumberg, K. B. Towles, J. F. Beausang, P. C. Nelson, R. Phillips, Concentration and length dependence of dna looping in transcriptional regulation, PloS one 4 (5) (2009) e $5621-\mathrm{e} 5621$.

[62] S. Johnson, M. Lindén, R. Phillips, Sequence dependence of transcription factor-mediated dna looping, Nucleic acids research (2012) gks473.

[63] D. G. Priest, L. Cui, S. Kumar, D. D. Dunlap, I. B. Dodd, K. E. Shearwin, Quantitation of the dna tethering effect in long-range dna looping in vivo and in vitro using the lac and $\lambda$ repressors, Proceedings of the National Academy of Sciences 111 (1) (2014) 349-354.

[64] Y.-J. Chen, S. Johnson, P. Mulligan, A. J. Spakowitz, R. Phillips, Modulation of dna loop lifetimes by the free energy of loop formation, Proceedings of the National Academy of Sciences 111 (49) (2014) $17396-17401$.

[65] P. J. Mulligan, Y.-J. Chen, R. Phillips, A. J. Spakowitz, Interplay of protein binding interactions, dna mechanics, and entropy in dna looping kinetics, Biophysical Journal 109 (3) (2015) 618-629.

[66] A. Esadze, J. Iwahara, Stopped-flow fluorescence kinetic study of protein sliding and intersegment transfer in the target DNA search process, Journal of Molecular Biology 426 (1) (2014) 230-244.

[67] A. Esadze, C. A. Kemme, A. B. Kolomeisky, J. Iwahara, Positive and negative impacts of nonspecific sites during target location by a sequence-specific DNA-binding protein: origin of the optimal search at physiological ionic strength, Nucleic Acids Research 42 (11) (2014) 7039-7046.

[68] O. G. Berg, M. Ehrenberg, Association kinetics with coupled three- and one-dimensional diffusion, Biophysical Chemistry 15 (1) (1982) 41-51.

[69] N. P. Stanford, One- and three-dimensional pathways for proteins to reach specific DNA sites, The EMBO Journal 19 (23) (2000) 6546-6557.

[70] J. S. Graham, R. C. Johnson, J. F. Marko, Concentration-dependent exchange accelerates turnover of proteins bound to double-stranded dna, Nucleic acids research 39 (6) (2011) 2249-2259. 